\title{
Single nucleotide polymorphism array analysis of microsatellite-stable, diploid/near-diploid colorectal carcinomas without the $\mathrm{CpG}$ island methylator phenotype
}

\author{
MICHAEL LINNEBACHER ${ }^{1}$, CHRISTIANE OSTWALD ${ }^{2}$, DIRK KOCZAN ${ }^{3}$, TAREQ SALEM $^{2}$, \\ BJÖRN SCHNEIDER ${ }^{2}$, MATHIAS KROHN ${ }^{1}$, MATHIAS ERNST ${ }^{4}$ and FRIEDRICH PRALL ${ }^{2}$ \\ ${ }^{1}$ Department of Surgery and Institutes of ${ }^{2}$ Pathology, ${ }^{3}$ Immunology and ${ }^{4}$ Biostatistics and Informatics \\ in Medicine and Ageing Research, University of Rostock, D-18055 Rostock, Germany
}

Received July 17, 2012; Accepted October 10, 2012

DOI: $10.3892 / \mathrm{ol} .2012 .1006$

\begin{abstract}
Colorectal carcinomas are considered to progress by chromosomal instability (CIN), or microsatellite instability (MSI) and/or epigenetic gene silencing; however, in previous studies we observed a small fraction of tumours without this molecular phenotype. To further investigate these 'X-type' tumours, neoplastic glands from five tumours were isolated by laser-capture microdissection and used for single nucleotide polymorphism (SNP) array analyses. DNA from our own lowpassage primary colorectal carcinoma cell lines $(n=9)$ was used for comparison. Two of these 'X-type' tumours had very low numbers of aberrations (totals of four and five, respectively), consisting of trisomies and arm amplifications. Conversely, aberrations were markedly more frequent in the control cases and three of the 'X-type' tumours (range, 11-40). These aberrations included deletions of chromosomes and chromosome arms, uniparental disomies (UPD), trisomies and arm amplifications. Recurrent microdeletions $(<1 \mathrm{MB})$ were observed at 3p14.2 (FHIT), 16p13.2 (A2BP1) and 20p12.1 (MACROD2). Microsatellite analyses with polymorphic markers at five 'canonical' colorectal carcinoma loci demonstrated a complete loss of one allele in all but one case. When compared to the SNP arrays, concordant results were observed in $93 \%$ of tests; however, this was only if DNA from cell lines or laser-capture microdissections was used. In conclusion, colorectal carcinomas may develop without the classic molecular features of CIN, MSI and/or CpG island methylator phenotype (CIMP), but this is a rare event. UPD is frequent but does not define a separate molecular phenotype. Furthermore, our study supports the notion that SNP arrays are reliable for genomewide detection of deletions and UPD, but discourages the use
\end{abstract}

Correspondence to: Professor Friedrich Prall, Institute of Pathology, University of Rostock, Strempelstraße 14, D-18055 Rostock, Germany

E-mail: friedrich.prall@med.uni-rostock.de

Key words: SNP array, colorectal carcinoma, loss of heterozygosity of microsatellite analyses to detect loss of heterozygosity with DNA from whole tissues.

\section{Introduction}

Currently, chromosomal instability (CIN) and microsatellite instability (MSI) are considered to be the principal driving forces of carcinogenesis (1). In the majority of cases these are mutually exclusive, but epigenetic gene silencing can act as an additional factor to either (2). Thus, driven by chromosomal instability, cancers are thought to progress when gene functions are compromised by loss of chromosomes or larger parts thereof, by deletions/insertions of a small number of nucleotides within microsatellites located in the coding regions of genes or by methylation of cytosines in or near gene promoters, leading to loss of gene expression. Each of these carcinogenic driving forces is reflected in a molecular phenotype of cancers that can be readily assessed in a molecular pathology laboratory. Specifically, DNA cytophotometry and/or allelotyping with polymorphic microsatellite markers is used to test for CIN; microsatellite analysis of markers particularly susceptible to instability (usually including the Bethesda markers) (3) is used to address MSI, and methylation-specific PCR (ideally with quantitative approaches such as MethyLight) (4) is used to assess whether promoter methylation is frequent in a cancer (the $\mathrm{CpG}$ island methylator phenotype, CIMP). For colorectal carcinoma, these molecular features underlie a recently proposed 'molecular classification' (5).

In a previous study (6), we described the application of such molecular assays to a larger consecutive series of colorectal carcinomas $(n=130)$. Eleven cases were observed that were not wholly concordant with our predictions and the current view regarding carcinogenesis. These tumours were diploid by DNA flow cytometry and no evidence of allelic imbalance at any of the 'canonical' loci of colorectal carcinoma (5q21, 8p21, 9p21, 17p13 and 18q21) was observed. Additionally, they were microsatellite-stable and lacked evidence of CIMP when a MethyLight marker panel was used. We proposed that this unexpected molecular phenotype may be indicative of an unusual carcinogenic pathway, and suggested the tentative designation of 'X-type' tumours. 
As was discussed in our previous study (6), technical limitations of the analyses may have influenced the results. Notably, DNA cytophotometry is prone to omit genomic losses if diploidy is maintained. This happens in uniparental disomy, a molecular feature researchers are increasingly becoming aware of $(7,8)$. Furthermore, small amplifications or deletions in loci not targeted by our microsatellite marker panel would have escaped detection by both allelotyping and DNA cytophotometry. Finally, DNA was extracted from whole tissues in our previous study (6). Although the tumour-stroma ratio had been controlled for by microscopic examination of scout slides, the possibility of false-negative identifications of allelic imbalance remained.

It may be expected that these technical limitations are overcome when single nucleotide polymorphism (SNP) array analysis is conducted with tumour tissue separated from the stroma by laser-capture microdissection. SNP array analysis simultaneously interrogates the entire genome of a tumour for copy number and allele status. Additionally, by laser-capture microdissection, confounding effects of admixed non-tumour DNA are avoided. Using this approach, as a first objective of this study, we further investigated the 'X-type' colorectal carcinomas and used DNA from several of our primary colorectal carcinoma cell lines of various molecular phenotypes for comparison. As a second objective of methodological interest, this approach enabled a direct comparison of results from SNP array and microsatellite analyses.

\section{Materials and methods}

Prior written informed consent was obtained from all patients, and all procedures were approved by the Ethics Committee of the University of Rostock (reference number II HV 43/2004) in accordance with generally accepted guidelines for the use of human material.

Tumour tissues and primary colorectal carcinoma cell lines. Tumour tissue of sufficient quantity was available for five of the original 11 'X-type' tumours (6); these five cases were used in this study. Neoplastic glands were separated from the stroma by laser-capture microdissection using a PALM laser-capture microdissection device (Carl Zeiss, Göttingen, Germany).

For comparison, we selected nine of our primary colorectal carcinoma cell lines from early passages. Molecular typing of these control cases was implemented as described (6). Clinicopathological and molecular data of the tumours included in this study are summarized in Table I.

Patients' normal mucosa (for 'X-type' tumours) or B-lymphocytes (for cell lines) were used to obtain individually matched non-tumour DNA.

DNA extraction and SNP array hybridisation. Laser-capture microdissected sample material was incubated with $8 \mu \mathrm{l}$ proteinase $\mathrm{K}(20 \mathrm{mg} / \mathrm{ml})$ overnight at $56^{\circ} \mathrm{C}$. DNA was subsequently purified using the column-based NucleoSpin Tissue XS kit (Macherey-Nagel, Düren, Germany), according to the manufacturer's protocol for microdissected material.

The DNA samples were treated as described in the Affymetrix Cytogenetics Copy Number Assay User Guide; $500 \mathrm{ng}$ genomic DNA sample was portioned into two aliquots of $250 \mathrm{ng}$ and these were cleaved by restriction endonucleases (StyI and NspI). Following adapter ligation, a reduction of the genomic complexity was performed by limited cycle preparative PCR. In alteration to the protocol, the PCR products were cleaned up by an ultrafiltration procedure using NucleoFast 96 PCR Plates (Macherey-Nagel). Fragmentation by DNaseI and end labelling was conducted using the Genome-Wide Human Nsp/Sty 5.0/6.0 Assay kit (Affymetrix, Santa Clara, CA, USA). The hybridisation of Genome-Wide Human SNP 6.0 arrays was followed by $16-17$ hours incubation at $50^{\circ} \mathrm{C}$ in the GeneChip Hybridization Oven 640. After washing, staining and antibody amplification using the Fluidics Station 450, the SNP 6.0 arrays were scanned with the Affymetrix GeneChip Scanner 3000 (7G).

Processing of SNP array hybridisation data and evaluation. For copy number and loss of heterozygosity ( $\mathrm{LOH})$ analysis, the intensity data files obtained by scanning of the processed microarrays (CEL files), were imported into the Genotyping Console Software (Affymetrix). Data processing followed the implemented standard workflow for SNP 6.0 array and unpaired analysis was performed using an implemented HapMap sample set of 270 individuals as a reference.

For visualisation, the result files were loaded into the Affymetrix GTC browser software to display the $\log 2$ ratio, copy number state and LOH state over a RefSeq track. The displays of tumour DNA hybridisations were directly compared with SNP array hybridisations of patients' normal DNA, chromosome by chromosome in each case. Aberrations of ploidy status and allele status were recorded under the following categories: i) loss or amplification of whole chromosomes or chromosome arms; ii) uniparental disomy or uniparental polysomy of whole chromosomes or chromosome arms; iii) subchromosomal deletions or amplifications, the chromosomal sites of which were recorded by reference to the chromosome bar at the bottom of the viewer, and the cut-off between extensive deletion/amplification versus microdeletions/microamplifications (see below) was arbitrarily set to $1 \mathrm{MB}$ of DNA; iv) subchromosomal uniparental disomy and v) microdeletions or microamplifications.

Microsatellite analyses. The microsatellite markers employed for allelotyping targeted the 'canonical' colorectal carcinoma loci $5 \mathrm{q} 21,8 \mathrm{p} 21,9 \mathrm{p} 21,17 \mathrm{p} 13$ and 18q21; technical details of these assays are described in our previous study (6). Two dinucleotide markers located at 3p14.2 (D3S1234, D3S1300) were added in the present study. For the control cases, the majority of microsatellite analyses were conducted using DNA from the cell lines; only in a minority of cases was DNA from corresponding xenografts used (details of the xenografting procedures have been published previously) (9). Microsatellite instability was tested with the Bethesda markers.

\section{Results}

Genomic aberrations recorded by SNP array analysis. SNP array hybridisations were performed successfully with genomic DNA extracted from the primary colorectal carcinoma cell lines and with DNA from laser-capturemicrodissected neoplastic glands of the colorectal carcinoma 
Table I. Summary of clinicopathological and molecular data of the cases included in the study.

\begin{tabular}{|c|c|c|c|c|c|c|}
\hline Tumour ID & Type & Site & Diameter $(\mathrm{cm})$ & TNM & Molecular type & Passage \\
\hline HROC 18 & $\mathrm{ADC}$ & Right colon & 4.0 & G2pT3pN0cM0 & spSTD & 13 \\
\hline HROC 24 & $\mathrm{ADC}$ & Right colon & 2.5 & G2pT2pN0cM0 & spMSI & 4 \\
\hline HROC 32 & $\mathrm{ADC}$ & Right colon & 5.0 & $\mathrm{G} 2 \mathrm{pT} 4 \mathrm{pN} 2 \mathrm{cM} 0$ & spSTD & 7 \\
\hline HROC 39 & $\mathrm{ADC}$ & Right colon & 10.0 & G3pT4pN0cM0 & spSTD & 8 \\
\hline HROC 40 & $\mathrm{ADC}$ & Left colon & 6.0 & G3pT3pN1cM0 & CIMP & 3 \\
\hline HROC 43 & $\mathrm{ADC}$ & Right colon & 5.0 & G3pT3pN2cM0 & spSTD & 4 \\
\hline HROC 46 & $\mathrm{ADC}$ & Right colon & 6.0 & G3pT3pN0cM1 & spSTD & 5 \\
\hline HROC 60 & $\mathrm{ADC}$ & Right colon & 3.2 & G2pT2pNOcM0 & CIMP & 6 \\
\hline HROC 69 & $\mathrm{ADC}$ & Right colon & 12.0 & G3pT3pN0cM0 & spSTD & 6 \\
\hline $\mathrm{T} 37$ & $\mathrm{ADC}$ & Left colon & 1.5 & G1pT1(sm3)pN0cM0 & 'X-type' & NA \\
\hline T53 & mucCa & Right colon & 12.0 & G1pT3pN0cM0 & 'X-type' & NA \\
\hline T97 & $\mathrm{ADC}$ & Rectum & 5.5 & G2pT3pN0cM0 & 'X-type' & NA \\
\hline T104 & $\mathrm{ADC}$ & Right colon & 8.0 & G2pT3pN0cM0 & 'X-type' & NA \\
\hline T109 & $\mathrm{ADC}$ & Rectum & 5.5 & G2pT2pN1cM0 & 'X-type' & NA \\
\hline
\end{tabular}

ADC, adenocarcinoma without specification; mucCa, mucinous carcinoma; right colon, caecum to splenic flexure; left colon, descending colon to sigmoid colon; diameter, maximum diameter of the tumour; spSTD, sporadic standard type; spMSI, sporadic microsatellite instability; CIMP, CpG island methylator phenotype; NA, not applicable.

Table II. Comparison of results from microsatellite (MS) and single nucleotide polymorphism (SNP) array analyses using DNA from colorectal carcinoma cell lines.

\begin{tabular}{lccr}
\hline & MS complete loss & MS incomplete loss & MS no loss \\
\hline SNP deletion & 37 & - & 2 \\
SNP UPD & 20 & - & 1 \\
SNP no aberration & 3 & 1 & 36 \\
\hline
\end{tabular}

Table III. Results of single nucleotide polymorphism (SNP) array and microsatellite (MS) analyses with DNA extracted from 'X-type' tumours after laser-capture microdissections.

\begin{tabular}{|c|c|c|c|c|c|c|c|c|c|c|}
\hline \multirow[t]{2}{*}{ ID } & \multicolumn{2}{|c|}{$5 q 21$} & \multicolumn{2}{|c|}{$8 \mathrm{p} 21$} & \multicolumn{2}{|c|}{$9 \mathrm{p} 21$} & \multicolumn{2}{|c|}{$17 \mathrm{p} 13$} & \multicolumn{2}{|c|}{$18 \mathrm{q} 21$} \\
\hline & SNP & MS & SNP & MS & SNP & MS & SNP & MS & SNP & MS \\
\hline T37 & - & - & - & - & - & - & - & - & - & - \\
\hline T53 & - & - & - & - & - & - & - & - & - & - \\
\hline T97 & - & - & - & - & UPD & $\mathrm{LOH}$ & DEL & $\mathrm{LOH}$ & UPD & $\mathrm{LOH}$ \\
\hline T104 & UPD & - & - & - & UPD & $\mathrm{LOH}$ & UPD & $\mathrm{LOH}$ & UPD & $\mathrm{LOH}$ \\
\hline T109 & - & - & - & - & - & - & DEL & - & UPD & $\mathrm{LOH}$ \\
\hline
\end{tabular}

Dash indicates absence of molecular aberations.

surgical specimens. All tumours harboured certain genomic aberrations. However, the frequency and type of aberration varied between cases.

Fig. 1A reveals the frequencies of all aberration types; very low total numbers were recorded for two of the 'X-type' tumours (T37 and T53, totals of five and four, respectively) and for HROC 24, which is of the sporadic MSI-type (a total of nine aberrations). The remaining tumours, including the three
'X-type' tumours T97, T104 and T109, exhibited higher totals (range, 11-40).

In Fig. 1B-D frequencies of different types of genomic aberrations are plotted per case. Fig. 1B reveals that deletions were numerous in the sporadic standard (spSTD)- and CIMPtype tumours (range, 8-26). Conversely, T97, T104 and T109 were observed to have uniparental disomies (UPD)/uniparental polysomies relatively frequently (range, 7-13). However, 
A

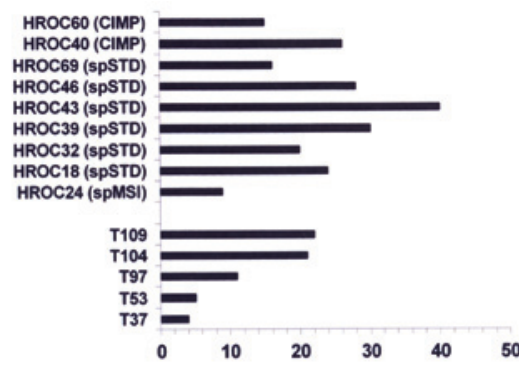

C

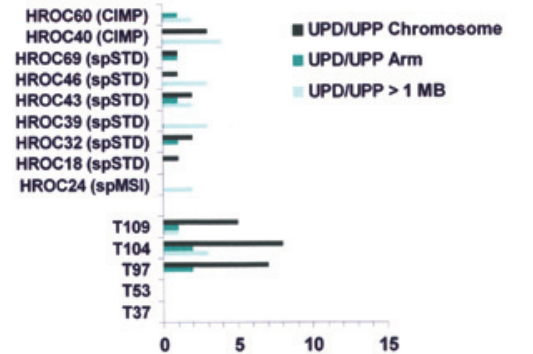

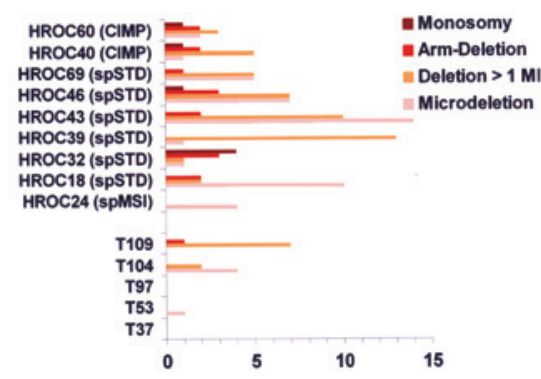

D

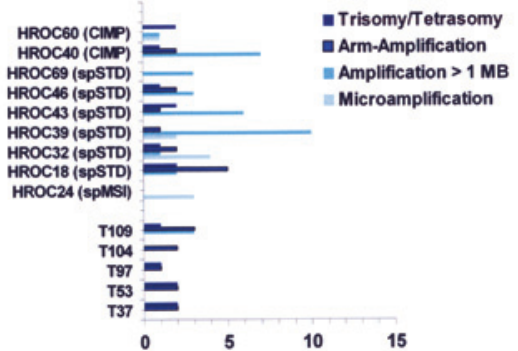

Figure 1. Frequencies and types of genomic aberrations recorded by single nucleotide polymorphism (SNP) array analysis and plotted per case. (A) Overall frequencies of aberrations; (B) frequencies of deletions; (C) uniparental disomies/polysomies; (D) amplifications.
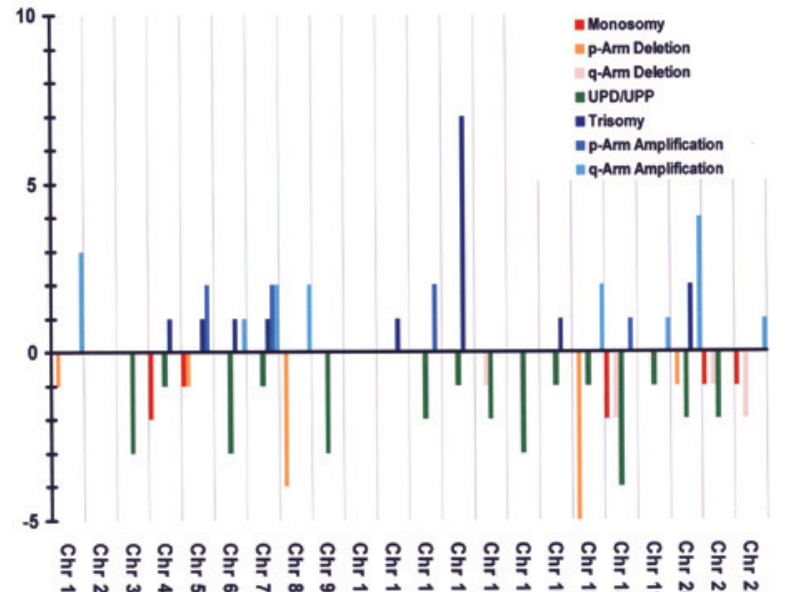

Figure 2. Frequencies and types of genomic aberrations recorded by single nucleotide polymorphism (SNP) array analysis and plotted per chromosome.

neither UPD nor uniparental polysomies were completely absent from the spSTD-/CIMP-type tumours, nor were deletions from the 'X-type' tumours. Notably, UPD/uniparental polysomies in the 'X-type' tumours T97, T104 and T109 most often affected whole chromosomes. Genomic aberrations in the other two 'X-type' tumours, T37 and T53, were trisomies or arm amplifcations (trisomies 13 and 16, and 8p-/20q arm amplifications for T37; trisomies 13 and 20, and 1q-/19q arm amplifications for T53).

All colorectal carcinoma cell lines had at least one microdeletion (range, 1-14) as did two of the 'X-type' tumours (T53 and T104; 1 and 4, respectively). Microdeletions were observed in the Affymetrix Genotyping Console Browser image as 'punched out' losses of hybridisation signals representing up to $1 \mathrm{MB}$ of DNA (but usually considerably less). Recurrent microdeletions were observed at 3p14.2 (5 of the colorectal carcinoma cell lines), 20p12.1 (4 of the colorectal carcinoma cell lines) and 16p13.2 (4 of the colorectal carcinoma cell lines and 2 of the 'X-type' tumours). The genes belonging to these loci were identified as FHIT, MACROD2 and A2BP1, respectively. The other microdeletions were distributed over the genome without any recognisable pattern.

In Fig. 2, the frequencies and types of genomic aberrations are plotted in relation to the different chromosomes. As can be observed from this figure, aberrations were most frequent in chromosomes 17,18 and 20.

SNP array analysis compared to allelotyping by microsatellite analysis. A comparative analysis was carried out with DNA from the nine colorectal carcinoma cell lines and/or xenografts, using 15 polymorphic microsatellite markers that represented 12 loci (as detailed in Materials and methods). Informative results were obtained in 100 of the 135 PCR reactions. Allelotyping was not possible due to the homozygosity of the microsatellite markers in 26 reactions, or not evaluable due to MSI in nine reactions (7 markers for HROC 24, which is of the spMSI-type, and 1 marker each for HROC 18 and HROC 69). Allelotyping showed loss of heterozygosity (LOH)/ allelic imbalance in 61 reactions. In all but one case, this was seen to involve complete loss of the allele in the electropherograms; LOH in its strict sense. As shown in Table II, there was overall concordance between the two methods, with discrepancies recorded for seven of the 100 analyses (7.0\%). Deletion or uniparental disomy, recorded by SNP array analysis, without evidence of allelic loss/allelic imbalance by microsatellite analysis was observed twice and once, respectively. Allelic loss or allelic imbalance, identified by microsatellite analysis, without evidence of genomic aberrations in the SNP arrays was recorded four times.

In our previous publication, 'X-type' tumours were defined by flow-cytometric diploidy, absence of MSI and absence of CIMP, in addition to absence of allelic imbalance at the canonical loci 5q21, 8p21, 9p21, 17p13 and 18q21. These analyses had been conducted using DNA from whole tissue cryostat sections with tumour fractions greater than $50 \%$ (6). 
Unexpectedly, for three of the tumours classified as 'X-type', the SNP arrays revealed UPD or deletion in nine of these loci. Therefore, allelotyping was then performed with DNA from laser-capture microdissections, as used for the SNP arrays. This showed allelic losses concordant with the SNP arrays in seven of the nine loci (details in Table III).

\section{Discussion}

The primary objective of this study was to further investigate the provisional molecular 'X-type' of colorectal carcinoma. Specifically, we addressed whether there would be evidence for, or at least an indication of, the regulating forces of carcinogenesis and tumour progression that differed from the orthodox triad of CIN, MSI and CIMP. Using SNP array analyses, we searched the entire genome for copy number changes and allelic losses, avoiding the potentially contaminating effects of non-neoplastic tissue by laser-capture microdissection. At present, only one study concerning colorectal carcinoma has been published that uses this approach (8).

Two of the 'X-type' tumours included in this study (T37 and T53) were markedly different from the rest of the cases; they differed from the remaining three 'X-type' tumours as well as from the control cases (Fig. 1). Apart from a single microdeletion observed in T53, these tumours did not have any allelic losses, neither by deletions nor by UPD. Thus, these two tumours appeared to be as similar as possible to an unorthodox molecular phenotype of colorectal carcinoma. Considering that they were selected from 130 colorectal carcinomas originally, this appears to be an exceedingly rare molecular phenotype. However, what makes these tumours unique and whether this molecular phenotype could have any pathogenetic implications are currently unknown. Notably, these colorectal carcinomas are at the borders of the usual clinicopathological spectrum, but in different ways to each other. T53 is a well-differentiated mucinous carcinoma without regional or distant metastases, but is well-advanced locally (diameter $12.0 \mathrm{~cm}$; pT3). This relatively unusual histotype may be the explanation for the unusual molecular phenotype. Conversely, T37 is a small cancer (diameter $2.5 \mathrm{~cm}$; pT1, though infiltrating well into the submucosa, sm3; node-negative), but is otherwise a morphologically non-descript adenocarcinoma with a moderate degree of tumour budding and with nuclear $\beta$-catenin translocation by immunohistochemistry (10). An explanation may be that this 'early' cancer simply did not have enough time to develop the load of genomic aberrations observed in the other tumours. The explanation for T37 may thus initially appear trivial; however, certain implications are evident. For example, it may be argued that if an 'early' colorectal carcinoma such as T37 is capable of sharing the phenotype of invasion with any other type of colorectal carcinoma (including metastasizing tumours) but does not share their molecular phenotype, then the function of the orthodox carcinogenic triad of CIN, MSI and CIMP may be a late effector in tumour progression, particularly in metastasizing disease. However, if invasiveness may be acquired without CIN, MSI or CIMP, and the pathogenetic function of this molecular phenotype is in tumour progression (the metastasizing course of the disease), then the relatively heavy loads of genomic loss observed in a number of our colorectal carcinomas without metastasis indicate that many of these may be functionally irrelevant. Such 'background noise' of genomic changes is a well-recognised phenomenon (11), but generalising from our observations, its extent and relevance in the interpretation of molecular analyses may be significantly underestimated. Moreover, it may transpire that it is extremely difficult to overcome this background noise in molecular studies, since for practical and ethical reasons, small cancers are significantly under-represented in tumour specimen collections, including in our own tumour bank.

However, for the remaining three tumours classified as 'X-type' (T97, T104 and T109), repeated microsatellite analyses with DNA from laser-capture microdissected tumour tissue was prompted by the SNP array analyses that had revealed UPD or deletions at certain loci tested in our previous study (Table III). These repeated microsatellite analyses revealed that $\mathrm{LOH}$ was indeed present at these loci in the majority of cases, having gone undetected in the initial tests with DNA from whole tumour tissues. Subsequently, these three tumours were reclassified as spSTD-type colorectal carcinoma, although UPD was a relatively frequent molecular feature of them and may explain their diploid status by DNA flow cytometry.

Our initial failure to detect allelic imbalance when allelotyping these three tumours raises an important methodological issue. The overwhelming majority of published LOH studies rely on DNA from whole tumour tissues. Typically, such as in our initial study, the tumour content is assessed by microscopic examination of histological sections, taken as sufficient at a fraction of $50-80 \%$ and $\mathrm{LOH}$ is scored if the tumour-normal ratios are below or above the arbitrary limits of 0.5 or 2.0, respectively. As noted previously (12), this microsatellite analysis of whole tissue DNA in reality is not a study of LOH but of allelic imbalance. Therefore, allelotyping is the correct designation for this procedure. As demonstrated in the present study, allelotyping carries a significant chance of false-negative determinations. Furthermore, as amplifications are not detected by this method, allelotyping is also prone to false-positive determinations. These methodical drawbacks strongly detract from the value of the majority of LOH studies of colorectal carcinomas or other solid tumours. LOH sensu strictu can only be diagnosed if, as was performed in this study, either tumour cell lines/xenografts or tumour tissue from laser-capture microdissections are used for the microsatellite analyses, and if complete absence of one allele is then observed.

Notably, in the majority of cases, microsatellite analyses at the loci tested in this study revealed a complete loss of one allele (Table II); an incomplete loss was recorded in only one instance. Thus, although deletion events or UPD may very often be background noise as has been discussed previously, they also have the potential to compromise gene function as proposed in the suppressor pathway concept. Therefore, when assessing the functional role of LOH by deletions or UPD in a given case, how these combine with mutations in the remaining alleles should be investigated. It has been demonstrated in colorectal carcinoma that gene mutations preferentially target a selection of a number of candidate cancer (CAN) genes, which are typically members of a signal transduction pathway that thereby undergoes dysregulation (13). The challenging task for researchers to negotiate is to find the relevant gene mutations by whole genome sequencing procedures, and then to compare them with 
the genome-wide allele status that can only be determined by SNP array analysis. In this context, the secondary objective of our study may be of methodological interest as follows.

As a secondary objective, the low-passage colorectal carcinoma cell lines of various molecular phenotypes that were included in this study allowed us to address how well LOH (by deletion or UPD) is represented in the SNP array analyses as compared with microsatellite analysis. If microsatellite analysis is informative and reveals a complete loss of one allele at a given locus, it may be regarded as a standard to compare with. Overall, discrepancies between microsatellite and SNP array analyses were observed in $7 \%$ of tests. Assuming microsatellite analysis is the standard, false-negative and false-positive determinations for LOH by SNP array analysis were observed in 4 and $3 \%$ of tests, respectively. We consider this to be a relatively low rate, attesting to the proficiency of the SNP array technique. However, it should be considered when interpreting data. To our knowledge, such comparisons have not been published and this may therefore be of interest for researchers who apply these techniques.

Microdeletions were a noteworthy observation in the SNP arrays. While the majority were distributed over the genome in no apparent order, recurrence was observed at three gene loci, viz., FHIT, MACROD2 and A2BP1, introducing the possibility of a potential functional role. In previous SNP array studies of colorectal carcinoma, deletions at 16p13.2 centering on A2BP1 have been described in a single study by Andersen et al (14). These authors also sequenced the gene in cases with deletions, failing to find mutations, and the deletions did not appear to correlate with differences of gene expression. Similiarly, correlative microsatellite/expression studies of FHIT did not reveal an effect of LOH on gene expression (15). Aberrations of the MACROD2 gene have not previously been identified for colorectal carcinoma; studies have only considered MACROD2 deletions in population-based genotyping (16). Therefore, though counterintuitive, there is no evidence to suggest that the 'punched out' gene losses so indicative of a functional role are implicated in the suppressor pathway of colorectal carcinoma. Alternatively, the recurrent loss of genetic material may arise as a consequence of recurrent translocation events that, as has been appreciated recently, are not as rare in solid tumours as previously thought (17). Notably, Andersen et al's study of interphase and metaphaseFISH for 16p13.2 using four commercial colorectal carcinoma cell lines for SW620 revealed a balanced translocation $(\mathrm{t}[3 ; 16])$ with the breakpoint centering on $16 \mathrm{p} 13$; for the other three cell lines, however, the deletions were interstitial deletions (14). Furthermore, rearrangements of the MACROD2, A2BP1 and FHIT genes in colorectal carcinoma were observed in a study by Bass et al (18), putatively due to structural fragility.

Taken together, in this study we have demonstrated that colorectal carcinomas may develop without the classic molecular features of CIN, MSI and/or CIMP, but this is a rare event. We observed that UPD is frequent in the context of CIN and most likely does not define a separate molecular phenotype. Furthermore, with regard to methodology, our study supports the notion that SNP array hybridisations are rather reliable for genome-wide detection of deletions and UPD, but strongly discourages $\mathrm{LOH}$ analyses with polymorphic microsatellite markers for DNA from whole tissues.

\section{Acknowledgements}

This study was in part supported by a grant (no. 108919) from the Deutsche Krebshilfe e.V.

\section{References}

1. Lengauer C, Kinzler KW and Vogelstein B: Genetic instabilities in human cancers. Nature 396: 643-649, 1998.

2. Toyota M, Ahuja N, Ohe-Toyota M, Herman JG, Baylin SB and Issa JP: CpG island methylator phenotype in colorectal cancer. Proc Natl Acad Sci USA 96: 8681-8686, 1999.

3. Boland CR, Thibodeau SN, Hamilton SR, Sidransky D, Eshleman JR, Burt RW, Meltzer SJ, Rodriguez-Bigas MA, Fodde R, Ranzani GN and Srivastava S: A National Cancer Institute workshop on microsatellite instability for cancer detection and familial predisposition: development of international criteria for the determination of microsatellite instability in colorectal cancer. Cancer Res 58: 5248-5257, 1998.

4. Ogino S, Cantor M, Kawasaki T, Brahmandam M, Kirkner GJ, Weisenberger DJ, Campan M, Laird PW, Loda M and Fuchs CS: $\mathrm{CpG}$ island methylator phenotype (CIMP) of colorectal cancer is best characterised by quantitative DNA methylation analysis and prospective cohort studies. Gut 55: 1000-1006, 2006.

5. Jass JR: Classification of colorectal cancer based on correlation of clinical, morphological and molecular features. Histopathology 50: 113-130, 2007.

6. Ostwald C, Linnebacher M, Weirich V and Prall F: Chromosomally and microsatellite stable colorectal carcinomas without the $\mathrm{CpG}$ island methylator phenotype in a molecular classification. Int J Oncol 35: 321-327, 2009.

7. Gaasenbeek M, Howarth K, Rowan AJ, Gorman PA, Jones A, Chaplin T, Liu Y, Bicknell D, Davison EJ, Fiegler H, Carter NP, et al: Combined array-comparative genomic hybridization and single-nucleotide polymorphism-loss of heterozygosity analysis reveals complex changes and multiple forms of chromosomal instability in colorectal cancers. Cancer Res 66: 3471-3479, 2006.

8. Andersen CL, Wiuf C, Kruhøffer M, Korsgaard M, Laurberg S and Ørntoft TF: Frequent occurrence of uniparental disomy in colorectal cancer. Carcinogenesis 28: 38-48, 2007.

9. Linnebacher M, Maletzki C, Ostwald C, Klier U, Krohn M, Klar E and Prall F: Cryopreservation of human colorectal carcinomas prior to xenografting. BMC Cancer 10: 362-371, 2010.

10. Prall F, Weirich V and Ostwald C: Phenotypes of invasion in sporadic colorectal carcinomas related to aberrations of the adenomatous polyposis coli (APC) gene. Histopathology 50: 318-330, 2007.

11. Tomlison IPM, Lambros MBK and Roylance RR: Loss of heterozygosity analysis: Practically and conceptually flawed? Genes Chromosomes Cancer 34: 349-353, 2002.

12. Devilee P, Cleton-Jansen AM and Cornelisse CJ: Ever since Knudson. Trends in Genet 17: 569-573, 2001.

13. Wood LD, Parsons DW, Jones S, Lin J, Sjöblom T, Leary RJ, Shen D, Boca SM, Barber T, Ptak J, Silliman N, et al: The genomic landscapes of human colorectal breast and colorectal cancers.Science 318: 1108-1113, 2007.

14. Andersen CL, Lamy P, Thorsen K, Kjeldsen E, Wikman F, Villesen P, Oster B, Laurberg S and Orntoft TF: Frequent genomic loss at chr16p13.2 is associated with poor prognosis in colorectal cancer. Int J Cancer 129: 1848-1858, 2011.

15. Wierzbicki PM, Adrych K, Kartanowicz D, Dobrowolski S, Stanislawowski M, Chybicki J, Godlewski J, Korybalski B, Smoczynski M and Kmiec Z: Fragile histidine triad (FHIT) gene is overexpressed in colorectal cancer. J Physiol Pharmacol S4: 63-70, 2009.

16. Bradley WE, Raelson JV, Dubois DY, Godin E, Fournier H, Privé C, Allard R, Pinchuk V, Lapalme M, Paulussen RJ and Belouchi A: Hotspots of large rare deletions in the human genome. PloS One 5: e9401, 2010.

17. Mitelman F, Johansson B and Mertens F: The impact of translocations and gene fusions on cancer causation. Nature Rev Cancer 7: 233-245, 2007.

18. Bass AJ, Lawrence MS, Brace LE, Ramos AH, Drier Y, Cibulskis K, Sougnez C, Voet D, Saksena G, Sivachenko A, Jing $\mathrm{R}$, et al: Genomic sequencing of colorectal adenocarcinomas identifies a recurrent VTI1A-TCF7L2 fusion. Nature Genetics 43: 964-968, 2011. 